\title{
Bunga Burrabugu - To Make Tomorrow
}

\author{
Annette Cairnduff \\ Social Inclusion, University of Sydney \\ compass.administration@sydney.edu.au
}

Keywords: Indigenous, higher education preparation, program design.

\section{Introduction}

Indigenous people are significantly underrepresented in higher education in Australia. In 2010 the Review of Higher Education Access and Outcomes for Aboriginal and Torres Strait Islander People (Berhendt Review) recommended that well-targeted university outreach programs could fill a number of gaps to address barriers to higher education. In response universities around the country have extended or developed programs to encourage and support pretertiary Indigenous students to consider higher education an option.

This paper will look at the design, implementation and evaluation elements of the approach taken at the University of Sydney, through its Compass Program, to support the preparation of Indigenous young people for higher education. It will provide a rationale for the program and background into the design approach, which has a strong focus of sustained engagement across years $7-12$ with Indigenous young people, their families, teachers and communities.

\section{Context}

The Berhrendt Review reported that although Indigenous peoples comprised $2.2 \%$ of the overall population they made up only $1.4 \%$ of student enrolments at universities, including only $1.1 \%$ of higher degree by research enrolments. (Berhrendt et al., 2010). Although there has been some increase in the number of Indigenous students participation in higher education in some years since then, the overall percentage of Indigenous students participating continues to sit at around 1\%. (Wilks et al., 2014). Indigenous students who do access higher education tend to be clustered into five areas of study: society and culture (32\%); health (19.9\%); education (17.6\%); management and commerce (11\%) and creative arts (7\%). (Wilks et al., 2014).

There are a number of barriers that hinder Indigenous students' access to higher education. Year 12 (or equivalent) completion is a significant issue. Despite some improvement in recent years the difference between Year 12 attainment for Indigenous and non-Indigenous students remains significant ${ }^{1}$. In 2013 the apparent ${ }^{2}$ retention rate of full-time Indigenous students from Years $7 / 8$ to Year 12 was $55 \%$ compared with $83 \%$ for non-Indigenous students. Indigenous students were retained from Year 11 to Year 12 at an apparent rate of $71 \%$ compared with $87 \%$ for non-Indigenous students. (AHMAC 2015 p. 88).

1. Indigenous student apparent retention rates from Year $7 / 8$ to Year 12 increased 55\% and Year $7 / 8$ to Year 10 20\% and Year 10 to Year $1230 \%$ between 1999 and 2013.

2. Apparent retention rates (ARR) are calculated based on aggregate enrolment data and provide an indicative measurement of student engagement in secondary education. The Department of Education and Early Childhood Development (DEECD) computes and publishes ARR data at a state-wide and DEECD region level only 
Low levels of numeracy and literacy continue to be a factor. The 2012 triennial OECD International Student Assessment indicated that 15-year-old Indigenous students were two years behind in reading, maths and scientific literacy. Without significant improvements in literacy and numeracy, a school-to-university pathway will continue to be inaccessible to the majority of Indigenous students.

Significant numbers of Indigenous students do not undertake the right units to get a university entrance score or rank (AHMAC 2015). Indigenous students are underrepresented in 'academic' courses in years 11/12 and in science and maths across all levels of education. High achievement during years 7 to 12 does not guarantee a transition into higher education for Indigenous people. The 2010 Longitudinal Survey of Australian Youth (Patton 2006) found that Indigenous students performing within the top brackets at school are not proportionately represented in university participation.

The barriers to higher education form a set of intertwined beliefs and circumstances which require active dismantling. Indigenous young people face a range of challenges including lack of confidence in their own academic ability; low expectations of academic achievement by teachers and career advisers; a lack of understanding among friends, family and their communities about the opportunities that higher education offers; and, a belief that higher education is not 'a place' for them a significant proportion of Indigenous students do not aim to attend higher education. A recent study into educational aspirations found that Australian students planning to remain at school until Year 12 or planning to participate in university at age 15 years are strong predictors of young people actually achieving these outcomes. (NVCER 2014) On a practical level, like many young people from low socio-economic backgrounds and other under-represented groups, Indigenous students will often be the first in their family to complete high school and so, like other first in family students, do not have ready access to information about pathways or a familial and cultural connection to higher education.

The Ministerial Council for Education, Early Childhood Development and Youth Affairs (MCEECDYA) recommended in the Aboriginal and Torres Strait Islander Education Action Plan $2010-2014$ the development of an action plan to close the gap in training, university and employment outcomes. Including "improved links between the school sector and the training, tertiary education and employment services sectors and support the development of innovative cross-sectoral approaches to programs and pathways for Aboriginal and Torres Strait Islander students." (p. 47) The MCEECDYA plan also recommended a review on how "new technologies can increase Aboriginal and Torres Strait Islander students' access to education and training."

\section{University Context}

The Wingara Mura - Bunga Burrabugu Strategy is the University's strategic commitment to valuing Indigenous culture and knowledges, building opportunity, capability and rights for Indigenous peoples. An important component of the strategy was to relocate the responsibility for action and implementation of activities to realise this commitment from the then centralised unit to across the whole of the University. Faculties and professional service units were resourced to develop local implementation plans.

The University already had a comprehensive, evidence informed and well-regarded program (Compass) that worked with school communities to encourage and prepare students from low socio-economic backgrounds and regional and remote areas for higher education and many of those schools had significant populations of Indigenous students. Compass was working with Indigenous pre-tertiary students in early high school on campus and through significant partnerships with the Australian Indigenous Mentoring Experience (AIME) and The Smith Family. 
Combined these factors allowed for the engagement of a broader group of resources (funding, staff and University student engagement, Faculty and professional service unit commitment and access to facilities) to support the scaling up of the activities to a suite of programs than had previously not been possible.

The programs that have been implemented to support Indigenous young people while they are still at school are an important element of the Wingara Mura Bunga Burrabugu Strategy's community engagement priority area. Although it is in in the early stages one of the immediate outcomes has been a direct link to and increase in student enrolment. It is understood that a program such as this would take time to build momentum and is a long-term commitment of the University to engage with Indigenous communities. Importantly this work has also contributed to developing cultural competence in both explicit, intentional ways and implicitly in our staff and students which is a core focus of the Strategy.

The University is working to introduce ideas of cultural competence firstly from the standpoint of Indigenous cultures through innovative learning, teaching, research and engagement. These broader efforts have been complemented by the development of a graduate attribute relating to cultural competence and the collaborative input into the delivery, content and teaching of the Compass program directly engaging staff with pre-tertiary Indigenous students.

The development and implementation of the programs for young people provides opportunities for staff both professional and academic, and University student ambassadors/mentors to develop their cultural competence ${ }^{3}$. This development is undertaken through formal 'training' sessions/workshops, online modules and informally through the engagement of local Indigenous people and staff as Aunties and Uncles to advise and support staff, University students and participants throughout the development and implementation of the programs.

The formal training, undertaken online and in workshops encourages and supports those involved to build their personal capabilities and cultural understandings and ensure a safe and respectful environment is provided for our young program participants. Our training encourages the staff and University students involved to become more aware of themselves - to think about their thinking - in relation to their interactions with people of different cultures, in particular Indigenous peoples. The key goal is becoming aware of inbuilt prejudices and preferences and reflecting on the potential differences between what you think you do and what you actually do. Elements of this can be confronting, thinking and reflecting on how we see each other, the assumptions and stereotypes, power dynamics, the social influences that contribute to discrimination - even when we are not aware of it.

During the on-campus programs Aunties and Uncles play an important role in supporting staff and University-students to build their cultural competence and ensuring a culturally safe space for the young participants. Aunties and Uncles, recruited from the University and local community provide culturally and age appropriate guidance and advice to the Indigenous participants on the programs and supporting staff and University students through constructive feedback, advice to support positive engagement and the participants wellbeing. Our Aunties and Uncles are available throughout the program activities. They are visible presence attending formal debriefing and handover sessions as well as being around during meal, evening and social times.

3. Cultural competence is a broad and malleable concept. There is no one definition, however in 1989 Cross et, al offered a definition that has provided a foundation for adaption and modification in different purposes and contexts. Cultural competence is a set of congruent behaviours, attitudes and policies that come together in a system, agency or among professionals; enabling that system, agency or those professionals to work effectively in cross-cultural situations (Cross et al, 1989). 
The combination of both 'formal' and 'informal' opportunities for those people working on the program has proved to be a potent one with University students who work on the programs as 'ambassadors' (taking a 'group leader' role with participants during on-campus activities) reporting in the post session surveys an increased and deeper understanding of Indigenous and Torres Strait issues and cultures and that they feel more confident and equipped to be 'culturally responsive' when working with Indigenous young people. For academic staff there is often an acknowledgment of the huge gap in knowledge, or deficit that they have about Indigenous history and culture, and an impetus to expand their understanding with subsequent training.

\section{Program Design \& Evaluation}

The design of the program builds upon the evidence that multi-faceted, sustained and early engagement has the most impact on academic preparation, confidence, aspiration and motivation for higher education. (Gale et al 2010, Sauders et al 2013, Bekley et al 2015).

Some of the University components of the program include in-school and on campus activities, two residential programs introducing students to life on campus, homework clubs, parents days, online activities and access to academic support. The University's programs are purposefully built and implemented as collaborations between non-Government agencies, schools, the University and young people themselves. The program intentionally brings together nonGovernment and school partners that support young Indigenous people through a mechanisms such as mentoring, tutoring, school capacity building, sporting teams with and aim to building their success in education. By engaging various local and community partners we ensure that there is reinforcement from a variety of sources of the messages about the opportunities for pathways to higher education.

The design process is facilitated through the implementation of a developmental evaluation process, which provides structured and on-going feedback during the development, implementation and evaluation stages. Developmental Evaluation is an evaluation approach that is designed to assist social change initiatives in complex environments. The approach is sometimes likened to the role of research and development in the private sector product development process because it facilitates real-time, or close to real-time, feedback to program staff thus facilitating a continuous development loop.

Patton (2010) describes developmental evaluation as a framework that:

"supports innovation development to guide adaptation to emergent and dynamic realities in complex environments. Innovations can take the form of new projects, programs, products, organizational changes, policy reforms, and system interventions. A complex system is characterized by a large number of interacting and interdependent elements in which there is no central control. Patterns of change emerge from rapid, real time interactions that generate learning, evolution, and development - if one is paying attention and knows how to observe and capture the important and emergent patterns. Complex environments for social interventions and innovations are those in which what to do to solve problems is uncertain and key stakeholders are in conflict about how to proceed."

This approach of continuous feed back and working collaboratively has allowed us to build on the strengths of each of the partners and focus on enhancing the depth of engagement with Indigenous young people to build an integrated, sustained and multi-layered approach 
to improving educational outcomes. The programs actively seeks feedback through a series of avenues including; a steering committee, pre and post feedback sessions with Faculty staff and students, debrief sessions with teachers and schools and formal (in some programs daily) surveys of participants and attending support staff. The input and reflection from these processes has driven the development of all of the program elements including the how (mode and style of delivery) and what (program content) undertaken as part of the program.

Over the course of the last few years this reflection has seen the programs grow and develop so that they begin early (in some cases as early as primary school); are sustained (students are consistently engaged from years $7-12$ ); multi-focussed on activities that build both personal and academic skills (academic support, enriched learning opportunities, personal confidence and motivation, awareness of career options, study skills, scholarship and pathway application writing skills and; multi-modal (online, on-campus, in school). This reflection and input has, importantly, led to the centrality of a strengths based approach. The programs actively acknowledge and celebrate the strengths and achievements of the young people involved. The programs focus on both their efforts and outcomes while working to build their sense of belonging and connection to further education and confidence to make informed decisions about their lives and know that there is support available.

A genuine commitment to this approach of engagement and feedback with stakeholders, participants and partners through structured and informal feedback has strengthened the capacity of the programs to meet the needs of the students involved. It has also contributed to the strong sense of ownership and connection that the young people and the schools and nonGovernment organisations involved have to the outcomes and the program itself. This is most clearly demonstrated by the 'repeat' engagement of the young people and their teachers in the activities and programs and also very visible in the feedback from participants who articulate that they can see the impact of their input on elements of the programs delivery or content.

\section{Program Innovation}

The focus on a continuous evaluation process enables responsive design that informs program development and encompasses input from all stakeholders (Aunties, Uncles, Indigenous staff and students from across the University, our school and NGO partners and the participants themselves). This active engagement takes the form of structured feedback (daily and pre and post of program evaluations, debriefs with partners) and less formal feedback from key stakeholders and a commitment to responsive design has allowed for new ideas to be tested and developed. One of the most innovative results of this has been the development of an online network, which works to support, inspire and engage the students involved in the oncampus and in-school programs when they return to their school communities and 'day to day' lives.

A unique tool (A.STAR) ${ }^{4}$ personalises interaction between higher education and young people through individually focussed and tailored online support and information. The network works to maintain the connection with each other and significant personal shifts that participants experience in the on-campus programs. The network (A.STAR) takes the form of an online magazine in which the contribution of program participants is a key aim. It provides an avenue for on going connection that links exploration of high school decisions, career and study options and preparation for higher education. Inspirational and informative it provides an avenue to connect students both to online academic support, each other and the program while they are not on-campus. A.STAR covers broad interests with arts, music, pop culture, science and sport. With articles on study, careers and pathways to university the magazine provides invaluable 
information on scholarships (including tips on how to apply) and the inside story on how to survive university in first year. Current university students write about their experiences of campus life and share insights from their chosen course and faculty. Alongside these stories A.STAR publishes the work of current high school students including articles, essays, opinion pieces, photography or videos. A.STAR staff are available to provide advice, feedback and edit student submissions.

A.STAR also provides students with the opportunity to understand and contribute to an arena of learning that is encapsulated within a digital platform. At the centre of this is an intrinsic engagement with digital literacy, narrative structures, visual and textual cultural representations. $A \cdot S T A R$ is also the gateway to access an online tutoring program that provides real-time and live homework assistance Sunday through to Thursday. Supporting young people to improve their academic outcomes and receive support in their homes from qualified teachers at the time they need it.

\section{Conclusion}

Indigenous people are significantly underrepresented in higher education in Australia. Welltargeted university outreach programs have an important role in addressing this gap and supporting pre-tertiary Indigenous students to consider higher education. This paper argues that the design process is an important element to ensure program success. A collaborative design, implementation and evaluation process allows for innovation and sustained and multi faceted engagement. Some crucial factors that have enabled this program to be as extensive as it is are: University leadership; fertile ground for change within a large organisation committed to effecting improved educational outcomes as a socio-cultural imperative; consistent strategic planning that enabled adequate resourcing and support for program implementation; and, the structuring of authentic partnerships that value collaborative practice. This approach of continuous feed back and working collaboratively has allowed us to build on the strengths of each of the partners and focus on enhancing the depth of engagement with Indigenous young people to build an integrated, sustained and multi-layered approach to improving educational outcomes.

\section{References}

Aboriginal and Torres Strait Islander Education Action Plan 2010 - 2014 Ministerial Council for Education, Early Childhood Development and Youth Affairs (MCEECDYA), and Education Services Australia. Retrieved from http://scseec.edu.au/site/DefaultSite/filesystem/documents/ATSI\%20 documents/ATSIEAP_web_version_final.pdf.

Australian Health Ministers' Advisory Council, 2015, Aboriginal and Torres Strait Islander Health Performance Framework 2014 Report, 2.05 Education outcomes for young people AHMAC. Retrieved from http://www.dpmc.gov.au/indigenous-affairs/publication/ aboriginal-and-torres-strait-islander-health-performance-framework-2014-report.

Behrendt, L., Larkin, S., Griew, R., \& Kelly, P. (2012). Review of higher education access and outcomes for Aboriginal and Torres Strait Islander people, final report. Retrieved from http://www.innovation. gov.au/highereducation/IndigenousHigherEducation/ReviewOfIndigenousHigherEducation/ FinalReport/lHERFinalReport.pdf

Beckley, A., Netherton, C. \& Singh, S. (2015). Closing the gap through Bridges to Higher Education. Research and Development in Higher Education: Learning for Life and Work in a Complex World, 38 (pp 416-435). Melbourne, Australia. 6 - 9 July 2015. 
Cross, T., Bazron, B., Dennis, K., \& Isaacs, M., (1989). Towards A Culturally Competent System of Care, Volume I. Washington, DC: Georgetown University Child Development Center, CASSP Technical Assistance Center.

Gale, T., Sellar, S., Parker, S., Hattam, R., Comber, B., Tranter, D. \& Bills, D. (2010). Interventions early in school as a means to improve higher education outcomes for disadvantaged (particularly low SES) students. Canberra: Department of Education, Employment and Workplace Relations. Australian Government.

Homel, J \& Ryan, C. (2014). Educational outcomes: the impact of aspirations and the role of student background characteristics. Adelaide, Australia: NCVER.

Moore, J., Sanders, J., Higham, L. (2013). Literature review of research into widening participation to higher education. Report to HEFCE and OFFA by ARC Network.

OECD (2015). "PISA: Programme for International Student Assessment", OECD Education Statistics (database). DOI: http://dx.doi.org/10.1787/data-00365-en (Accessed on 03 August 2015).

Patton, M.Q. (2006). Evaluation for the Way We Work. The Nonprofit Quarterly. Vol. 13 (1): 28-33. Retrieved via http://www.scribd.com/doc/8233067/Michael-Quinn-Patton-DevelopmentalEvaluation-2006 NCVER 2014, Longitudinal Surveys of Australian Youth annual report 2013. Adelaide, Australia: NCVER.

Wilks, J.L., \& Wilson, K. (2014). 'Can’t be what you can’t see': The transition of Aboriginal and Torres Strait Islander students into higher education. Literature Review 2014. Broome, Australia: University of Notre Dame Australia. Retrieved from http://www.nd.edu.au/_data/assets/pdf_ file/0018/122364/OLT-ID-SI11-2138-Lit-Rev-Final-14-March-2014.pdf. 